\title{
EXPONENTIALLY SMALL CORRECTIONS TO DIVERGENT ASYMPTOTIC EXPANSIONS OF SOLUTIONS OF THE FIFTH PAINLEVÉ EQUATION
}

\author{
F. V. Andreev and A. V. Kitaev
}

\begin{abstract}
We calculate the leading term of asymptotics for the coefficients of certain divergent asymptotic expansions for the solutions of the fifth Painlevé equation $\left(P_{5}\right)$ by using the isomonodromy deformation method and the Borel transform. Unexpectedly, these asymptotics appear to be periodic functions of the coefficients of $P_{5}$. We also show the relation of our results with some other facts already known in the theory of the Painlevé equations established by other methods: (1) a connection formula for the third Painlevé equation; (2) a condition for existence of rational solutions for $P_{5}$; (3) and a numerical study of the $\tau$ function for $P_{5}$.
\end{abstract}

\section{Instead of introduction}

There are several interesting asymptotic properties of the $\tau$-function associated with a particular one-parameter family of solutions of the third Painlevé equation $\left(P_{3}\right)$ with a special set of coefficients: this family can be also viewed as the fifth Painlevé transcendent for a very special choice of its parameters. Let us recall some of these properties, formulated in terms of the function $\sigma=\sigma(x ; \lambda)$, which is the solution of the following ordinary differential equation (ODE),

$$
\left(x \sigma^{\prime \prime}\right)^{2}=-16\left(x \sigma^{\prime}-\sigma\right)\left(x \sigma^{\prime}-\sigma+\frac{1}{4}\left(\sigma^{\prime}\right)^{2}\right),
$$

where $^{\prime}=\frac{d}{d x}$, satisfying the boundary condition

$$
\sigma(x ; \lambda) \underset{x \rightarrow 0}{=}-2 \lambda x-(2 \lambda x)^{2}-\ldots
$$

If $\lambda=\frac{1}{\pi}$, then

$$
\sigma\left(x ; \frac{1}{\pi}\right) \underset{x \rightarrow+\infty}{=}-x^{2}-\frac{1}{4}-\sum_{n=1}^{\infty} \frac{c_{n}}{x^{2 n}}+\mathcal{O}\left(x^{-\infty}\right),
$$

Received March 20, 1997.

1991 Mathematics Subject Classification. 34A20, 34E05, 30B40, 33E30, 40G10.

The first author was partially supported by the grant PRO-MATH and Soros Foundation under the grant ME-430. During the preparation of the final version of this paper the second author was supported by the Alexander von Humboldt Foundation. 
where

$$
c_{1}=\frac{1}{16}, c_{2}=\frac{5}{32}, c_{3}=\frac{131}{128}, c_{4}=\frac{6575}{512}, \ldots, c_{n}, n \geq 5
$$

can be obtained recursively by substituting expression (1.3) into Eq. (1.1).

The asymptotic series (1.3) diverges. E. L. Basor and C. A. Tracy [1] stated the following

Conjecture $^{1}$ :

$$
c_{n} \underset{n \rightarrow \infty}{\sim} \text { const } n !(n-1) !
$$

where const $\approx 0,10132$.

The function $\sigma(x ; \lambda)$ and its $\mathrm{m}$-th-order derivatives for $\lambda=\frac{1}{\pi}$ define the function $\left.E_{2}(m ; s)\right|_{s=\frac{\pi x}{2}}$, which is the probability that a randomly chosen interval of the length $s$ contains $m$ eigenvalues in the Gaussian Unitary Ensemble (see $[2],[3])$. We are going to investigate the function $E_{2}(m ; s)$ via the isomonodromy deformation method in a separate paper while here, we focus our attention on the calculation of the exponentially small correction term to the divergent expansion (1.3) and its intimate link with the asymptotics of the sequence $c_{n}$. We found that, among the functions $\sigma(x ; \lambda)$ with $\lambda>0$, only one, for $\lambda=\frac{1}{\pi}$, has the asymptotic expansion (1.3), (1.4) as $x \rightarrow \infty$, which is in agreement with the results obtained by a different method in [4]. Note that there is a one-parameter family of solutions of Eq. (1.1), other then $\sigma(x ; \lambda)$, which has asymptotics (1.3) as $x \rightarrow+\infty$. We calculated the explicit value of the const in (1.5): it is equal to

$$
\frac{1}{\pi^{2}}=
$$

$0,1013211836423377714438794632097276389043587746722465488 \ldots$.

Actually, in this work we consider a more general problem than that discussed above. Here, we investigate a one-parameter family of solutions of $P_{5}$ with the general set of coefficients which has (1.3)-like algebraic asymptotic expansion, so that, the results announced above are just a particular case of the ones obtained for $P_{5}$. As another result of our study we have found unexpected and interesting property of the coefficients of the divergent algebraic asymptotic expansions, which is general for all other Painlevé equations $\left(P_{2}-P_{5}\right)$ possessing the solutions with such expansions: This property is that the large $n$ asymptotics of the coefficients of the divergent asymptotic expansion are periodic functions of the coefficients of the Painlevé equations. Note that, being formally substituted into the Painlevé equations, the coefficients of the divergent algebraic series can be found via recurrence relations as polynomials of the coefficients of the Painlevé equations.

\footnotetext{
${ }^{1}$ As they told us later (June 1996, Mount Holyoke), this conjecture was based on a numerical study of the sequence $c_{n}$.
} 
In the next section, we recall some basic facts concerning the isomonodromy approach to $P_{5}$. Our results are formulated in Section 3, where we also give a new derivation of the theorem on classification of a special class of rational solutions of $P_{5}, y(x): y(x) \underset{x \rightarrow \infty}{\rightarrow}-1$, which was first proved in [5] and by other method in [6]. In section 4 , we apply the Borel transform to find the asymptotics of the coefficients of the divergent series for $P_{5}$. In Appendix, we consider two applications of our results to $P_{3}$ : (1) we prove asymptotic expansion $\left.(1.5), 1.6\right)$ and (2) verify that, for a very special choice of the coefficients of $P_{5}$, our result agree with the connection formula for $P_{3}$, which was proved in [7].

\section{The fifth Painlevé equation and its monodromy manifold}

Let us recall that, in [8], it was shown that isomonodromy deformations of the linear ODE

$$
\frac{d}{d \lambda} \Psi=\left(\frac{t}{2} \sigma_{3}+\frac{A_{0}(t)}{\lambda}+\frac{A_{1}(t)}{\lambda-1}\right) \Psi
$$

where $A_{k}(t) \in s l_{2}(\mathbb{C})$ and independent of $\lambda$, are governed by the following system of nonlinear ODEs:

$$
\begin{aligned}
& t \frac{d y}{d t}=t y-2 z(y-1)^{2}-(y-1)\left(\frac{\Theta_{0}-\Theta_{1}+\Theta_{\infty}}{2} y-\frac{3 \Theta_{0}+\Theta_{1}+\Theta_{\infty}}{2}\right) \\
& t \frac{d z}{d t}=y z\left(z+\frac{\Theta_{0}-\Theta_{1}+\Theta_{\infty}}{2}\right)-\frac{1}{y}\left(z+\Theta_{0}\right)\left(z+\frac{\Theta_{0}+\Theta_{1}+\Theta_{\infty}}{2}\right) \\
& t \frac{d}{d t} \log u=-2 z-\Theta_{0}+y\left(z+\frac{\Theta_{0}-\Theta_{1}+\Theta_{\infty}}{2}\right)+\frac{1}{y}\left(z+\frac{\Theta_{0}+\Theta_{1}+\Theta_{\infty}}{2}\right)
\end{aligned}
$$

where $\Theta_{\nu}(\nu=0,1, \infty)$ are complex constants - the parameters of the formal monodromy. Eliminating the function $z(t)$ from Eqs. (2.2) and (2.3), one finds that the function $y(t)$ satisfies $P_{5}$ :

$$
\begin{aligned}
\frac{d^{2} y}{d^{2} t}=\left(\frac{1}{2 y}+\frac{1}{y-1}\right)\left(\frac{d y}{d t}\right)^{2}-\frac{d y}{t d t}+ & \frac{(y-1)^{2}}{t^{2}}\left(\alpha y+\frac{\beta}{y}\right)+\frac{\gamma y}{t}+\frac{\delta y(y+1)}{y-1}, \\
\alpha & =\frac{1}{2}\left(\frac{\Theta_{0}-\Theta_{1}+\Theta_{\infty}}{2}\right)^{2}, \\
\beta & =-\frac{1}{2}\left(\frac{\Theta_{0}-\Theta_{1}-\Theta_{\infty}}{2}\right)^{2} \\
\gamma & =1-\Theta_{0}-\Theta_{1}, \delta=-\frac{1}{2} .
\end{aligned}
$$

The so-called $\tau$-function [8], [9] plays an important role in applications of $P_{5}$ :

$$
\frac{d}{d t} \log \tau_{p}(t) \equiv H_{p} \equiv-\frac{1}{2 t} \underset{\lambda=p}{\operatorname{Res}} \operatorname{tr} A^{2}(\lambda, t), p=0,1 ; H_{0}+H_{1}=\frac{\Theta_{\infty}}{2},
$$


where $A(\lambda, t)$ is the matrix in the parenthesis in Eq. (2.1). Using this definition it is easy to write an explicit representation of the $\tau$-function in terms of solutions $(y=y(t), z=z(t))$ of the system (2.2), (2.3) (see [8], [10]). It is also convenient to introduce an auxiliary function

$$
\zeta(t) \equiv \zeta\left(\Theta_{0}, \Theta_{1}, \Theta_{\infty} ; t\right) \equiv-t H_{1}+\frac{t}{2}\left(\Theta_{0}+\Theta_{\infty}\right)+\frac{\Theta_{0}}{2}\left(\Theta_{0}+\Theta_{\infty}\right)
$$

In particular, it is known [8] that the function $\zeta(t)$ solves the following ODE:

$$
\begin{aligned}
& \left(t \frac{d^{2} \zeta}{d t^{2}}\right)^{2}=\left(\zeta-t \frac{d \zeta}{d t}+2\left(\frac{d \zeta}{d t}\right)^{2}-\left(2 \Theta_{0}+\Theta_{\infty}\right) \frac{d \zeta}{d t}\right)^{2} \\
& -4 \frac{d \zeta}{d t}\left(\frac{d \zeta}{d t}-\Theta_{0}\right)\left(\frac{d \zeta}{d t}-\frac{1}{2}\left(\Theta_{0}-\Theta_{1}+\Theta_{\infty}\right)\right)\left(\frac{d \zeta}{d t}-\frac{1}{2}\left(\Theta_{0}+\Theta_{1}+\Theta_{\infty}\right)\right)
\end{aligned}
$$

which is for $\Theta_{0}=\Theta_{1}=\Theta_{\infty}=0$ and $t=4 \imath x$ coincide with Eq. (1.1).

The solutions of system (2.2)-(2.4) are in one-to-one correspondence with the points of the manifold of monodromy data $\mathcal{M}_{5}\left(\Theta_{0}, \Theta_{1}, \Theta_{\infty}\right)$. The detailed definition of $\mathcal{M}_{5}\left(\Theta_{0}, \Theta_{1}, \Theta_{\infty}\right)$ can be found in our previous works [10, 11]. Here, we recall that this manifold is defined by the matrix elements of the monodromy matrices $M_{0}$ and $M_{1}$, representing, respectively, the monodromy of the canonical solutions of Eq. (2.1) around the regular singularities at $\lambda=0$ and $\lambda=1$. Denoting the matrix elements of $M_{p}$ as $m_{i j}^{p}(p=0,1 ; i, j=1,2)$, we can define $\mathcal{M}_{5}\left(\Theta_{0}, \Theta_{1}, \Theta_{\infty}\right)$ by the following algebraic equations in $\mathbb{C}^{8}$ :

$$
\begin{gathered}
m_{11}^{0} m_{22}^{0}-m_{12}^{0} m_{21}^{0}=1, \quad m_{11}^{1} m_{22}^{1}-m_{12}^{1} m_{21}^{1}=1, \\
m_{11}^{0}+m_{22}^{0}=2 \cos \pi \Theta_{0}, \quad m_{11}^{1}+m_{22}^{1}=2 \cos \pi \Theta_{1}, \\
m_{11}^{1} m_{11}^{0}+m_{12}^{1} m_{21}^{0}=e^{-\pi \imath \Theta_{\infty}} .
\end{gathered}
$$

Note that, $\operatorname{dim}_{\mathbb{C}} M_{5}\left(\Theta_{0}, \Theta_{1}, \Theta_{\infty}\right)=3$. Stokes matrices, $S_{p+1}(p=0,1)$ (see [10] for definition), also play an essential role in the description of analytical properties of the solutions to Eq. (2.1). These matrices define the monodromy properties of the canonical solutions of Eq. (2.1) near the irregular singularity $\lambda=\infty$. The matrices $S_{p+1}$ have the following structure:

$$
S_{1}=\left(\begin{array}{cc}
1 & 0 \\
s_{1} & 1
\end{array}\right), \quad S_{2}=\left(\begin{array}{cc}
1 & s_{2} \\
0 & 1
\end{array}\right)
$$

where the complex parameters $s_{p+1}, p=0,1$ are called the Stokes multipliers. Further, we call $m_{i j}^{p}, s_{p+1}, p=0,1, i, j=1,2$ the monodromy coordinates. Although, it is easy to explicitly express $s_{p+1}$ in terms of $m_{i j}^{p}$, we further always write them down for the convenience of the reader.

The nonlinear system (2.2)-(2.4) has two singular points, $t=0$ and $\infty$, so that solutions of this system are defined on the universal covering of $\mathbb{C}\{0\}$. In the previous works $[10,11]$ we have investigated the asymptotics of the solutions of the system (2.2)-(2.4) as $t \rightarrow+0$ and $t \rightarrow+\infty$, that is why it is natural 
for us to consider the analytic continuation of these solutions from $(+0,+\infty)$. Furthermore, we understand the functions $y(t), z(t), u(t)$, and $\zeta(t)$, respectively, for $t \in \mathbb{C}$, as the ones analytically continued from $\mathbb{R}_{+}$. Since the system (2.2)(2.4) posses the Painlevé property, single-valued solutions can be defined on $\mathbb{C} \backslash[-0,-\infty)$. We call the ray $[-0,-\infty)$ the cut and denote as $y_{ \pm}(t), z_{ \pm}(t)$, $u_{ \pm}(t)$, and $\zeta_{ \pm}(t)$, respectively, the functions $y(t), z(t), u(t)$, and $\zeta(t)$ on the upper/lower sides of the cut, i.e., for $\arg t= \pm \pi$. Note that, the \pm -functions correspond to, i.e., define via Eq. (2.1), the same point of $\mathcal{M}_{5}\left(\Theta_{0}, \Theta_{1}, \Theta_{\infty}\right)$. Let us denote by $y^{-}(t), z^{-}(t), u^{-}(t)$, and $\zeta^{-}(t)$, respectively, a solution of system (2.2)-(2.4) and the corresponding function (2.7) with $\Theta_{\infty}$ changed to $-\Theta_{\infty}$ : The corresponding manifold of monodromy data is $\mathcal{M}_{5}\left(\Theta_{0}, \Theta_{1},-\Theta_{\infty}\right)$. As soon as the functions $y^{-}(t), z^{-}(t), u^{-}(t)$, and $\zeta^{-}(t)$, respectively, are known for real positive $t$ and an arbitrary point of $\mathcal{M}_{5}\left(\Theta_{0}, \Theta_{1},-\Theta_{\infty}\right)$, one can find the functions $y_{ \pm}(t), z_{ \pm}(t), u_{ \pm}(t)$, and $\zeta_{ \pm}(t)$ by using the following transformation,

$$
\begin{gathered}
u_{ \pm}(t)=\frac{1}{u^{-}\left(e^{\mp \pi \imath} t\right)}, \quad y_{ \pm}(t)=\frac{1}{y^{-}\left(e^{\mp \pi \imath} t\right)}, \\
z_{ \pm}(t)=-z^{-}\left(e^{\mp \pi \imath} t\right)-\Theta_{0}, \quad \zeta_{ \pm}(t)=\zeta^{-}\left(e^{\mp \pi \imath} t\right)+\Theta_{0} t+\Theta_{0} \Theta_{\infty},
\end{gathered}
$$

and the corresponding action of this transformation on the manifold of monodromy data:

$$
\begin{aligned}
& M_{p}^{+}=\sigma_{1} \hat{S}_{1} \hat{M}_{p} \hat{S}_{1}^{-1} \sigma_{1}, \quad S_{1}^{+}=\sigma_{1} e^{\pi \imath \Theta_{\infty} \sigma_{3}} \hat{S}_{2} e^{-\pi \imath \Theta_{\infty} \sigma_{3}} \sigma_{1}, \quad S_{2}^{+}=\sigma_{1} \hat{S}_{1} \sigma_{1} \\
& M_{p}^{-}=\sigma_{1} \hat{S}_{2}^{-1} \hat{M}_{p} \hat{S}_{2} \sigma_{1}, \quad S_{1}^{-}=\sigma_{1} \hat{S}_{2} \sigma_{1}, \quad S_{2}^{-}=\sigma_{1} e^{-\pi \imath \Theta_{\infty} \sigma_{3}} \hat{S}_{1} e^{\pi \imath \Theta_{\infty} \sigma_{3}} \sigma_{1}
\end{aligned}
$$

Here, $M_{p}^{ \pm}$and $S_{p+1}^{ \pm}(p=0,1)$ are the monodromy and Stokes matrices corresponding to the functions $y_{ \pm}(t), z_{ \pm}(t), u_{ \pm}(t)$, and $\zeta_{ \pm}(t), \hat{M}_{p}$ and $\hat{S}_{p+1}$ are the monodromy objects for $y^{-}(t), z^{-}(t), u^{-}(t)$, and $\zeta^{-}(t)$, respectively, i.e., their matrix elements define a point on $\mathcal{M}_{5}\left(\Theta_{0}, \Theta_{1},-\Theta_{\infty}\right)$. According the above definition, we have $M_{p}^{+}=M_{p}^{-}, S_{p+1}^{+}=S_{p+1}^{-}$. IT means that depending of what we want to ding: the + or, respectively, - functions, we have to take in Eqs. (2.9) and $(2.10)$, the solutions $y^{-}(\cdot), z^{-}(\cdot), u^{-}(\cdot)$, and $\zeta^{-}(\cdot)$ which correspond (in general case) to different points on $\mathcal{M}_{5}\left(\Theta_{0}, \Theta_{1},-\Theta_{\infty}\right)$, i.e., the matrices $\hat{M}_{p}$ and $\hat{S}_{p+1}$, in Eqs. (2.11) and (in general case) are different from those in Eqs. (2.12).

\section{Properties of truncated solutions}

Proposition 1. The system (2.2), (2.3) and Eq. (2.8) admit the following formal solutions:

$$
\begin{aligned}
& y_{F}(t)=-1+\sum_{n=1}^{\infty} \frac{\alpha_{n}\left(\Theta_{0}, \Theta_{1}, \Theta_{\infty}\right)}{t^{n}} \\
& z_{F}(t)=-\frac{t}{8}-\frac{2 \Theta_{0}+\Theta_{\infty}}{4}+\sum_{n=1}^{\infty} \frac{\beta_{n}\left(\Theta_{0}, \Theta_{1}, \Theta_{\infty}\right)}{t^{n}}
\end{aligned}
$$




$$
\begin{aligned}
\zeta_{F}(t)= & \frac{t^{2}}{16}+\left(2 \Theta_{0}+\Theta_{\infty}\right) \frac{t}{4}+\frac{3 \Theta_{0}^{2}}{4} \\
& +\frac{\Theta_{1}^{2}}{4}+\frac{\Theta_{\infty}^{2}}{4}+\frac{\Theta_{0} \Theta_{\infty}}{2}-\frac{1}{4}+\sum_{n=1}^{\infty} \frac{\gamma_{n}\left(\Theta_{0}, \Theta_{1}, \Theta_{\infty}\right)}{t^{n}}
\end{aligned}
$$

where the coefficients, $\alpha_{n}=\alpha_{n}\left(\Theta_{0}, \Theta_{1}, \Theta_{\infty}\right), \beta_{n}=\beta_{n}\left(\Theta_{0}, \Theta_{1}, \Theta_{\infty}\right)$, and $\gamma_{n}=\gamma_{n}\left(\Theta_{0}, \Theta_{1}, \Theta_{\infty}\right)$, are uniquely determined polynomials of their arguments of the degrees:

$$
\begin{aligned}
\operatorname{deg} \alpha_{n}\left(\Theta_{0}, \Theta_{1}, \Theta_{\infty}\right) & =n, \operatorname{deg} \beta_{n}\left(\Theta_{0}, \Theta_{1}, \Theta_{\infty}\right) \\
& =n+1, \operatorname{deg} \gamma_{n}\left(\Theta_{0}, \Theta_{1}, \Theta_{\infty}\right)=n+2 .
\end{aligned}
$$

Proof. The proof of Proposition 1 is just the straightforward substitution of the expansions (3.1), (3.2) and (3.3) into the system (2.2), (2.3) and Eq. (2.8) correspondingly. As the result one finds recurrence relations for the coefficients $\alpha_{n}, \beta_{n}$, and $\gamma_{n}$, which enable one to make the conclusions stated above.

Remark 1. The recurrence relations are rather complicated. We don't use them directly and that is why we don't write them down here. The following is the list of the few first polynomials :

$$
\begin{aligned}
\alpha_{1}= & 4\left(\Theta_{0}+\Theta_{1}-1\right), \quad \alpha_{2}=8 \Theta_{\infty}\left(\Theta_{1}-\Theta_{0}\right)-8\left(\Theta_{0}+\Theta_{1}-1\right)^{2}, \\
\alpha_{3}= & 16\left(\Theta_{0}+\Theta_{1}-1\right)\left(\Theta_{0}-\Theta_{1}+\Theta_{\infty}\right)^{2} \\
& +16\left(\Theta_{0}+\Theta_{1}-3\right)\left(\Theta_{0}+\Theta_{1}\right)^{2}+32\left(\Theta_{0}+\Theta_{1}\right), \\
\beta_{1}= & 0, \beta_{2}=\Theta_{\infty}\left(\Theta_{1}^{2}-\Theta_{0}^{2}\right), \beta_{3}=2\left(\Theta_{1}^{2}-\Theta_{0}^{2}\right)^{2}+2\left(\Theta_{\infty}^{2}-1\right)\left(2 \Theta_{0}^{2}+2 \Theta_{1}^{2}-1\right), \\
\gamma_{1}= & \beta_{2}, \quad \gamma_{2}=\frac{1}{2} \beta_{3}, \quad \gamma_{3}=4 \Theta_{\infty}\left(\Theta_{1}^{2}-\Theta_{0}^{2}\right)\left(2 \Theta_{0}^{2}+2 \Theta_{1}^{2}+\Theta_{\infty}^{2}-5\right), \\
\gamma_{4}= & 8\left(\Theta_{0}^{6}+\Theta_{1}^{6}-\Theta_{0}^{2} \Theta_{1}^{4}-\Theta_{1}^{2} \Theta_{0}^{4}+\Theta_{0}^{2} \Theta_{\infty}^{4}+\Theta_{1}^{2} \Theta_{\infty}^{4}+5 \Theta_{1}^{4} \Theta_{\infty}^{2}+5 \Theta_{0}^{4} \Theta_{\infty}^{2}\right. \\
& -2 \Theta_{0}^{2} \Theta_{1}^{2} \Theta_{\infty}^{2}-7 \Theta_{0}^{4}-7 \Theta_{1}^{4}-\frac{1}{2} \Theta_{\infty}^{4}-12 \Theta_{1}^{2} \Theta_{\infty}^{2}-12 \Theta_{0}^{2} \Theta_{\infty}^{2}+6 \Theta_{0}^{2} \Theta_{1}^{2} \\
& \left.+11 \Theta_{0}^{2}+11 \Theta_{1}^{2}+\frac{11}{2} \Theta_{\infty}^{2}-5\right) .
\end{aligned}
$$

Although it is not evident from the definition (2.7), one proves that $\zeta^{\prime}(t)=-z(t)$, which means that $\beta_{n+1}\left(\Theta_{0}, \Theta_{1}, \Theta_{\infty}\right)=n \gamma_{n}\left(\Theta_{0}, \Theta_{1}, \Theta_{\infty}\right)$. Another property of the polynomials $\alpha_{n}, \beta_{n}$, and $\gamma_{n}$ is that they can be rewritten as the polynomials of the coefficients $\alpha, \beta$, and $\gamma$ (see Eq. (2.6)) of $P_{5}$ (2.5), without any square roots! It is more or less clear for the polynomials $\alpha_{n}$, since they can be obtained recursively: by substituting expansion (3.1) into the Eq. (2.5), but than, the polynomials $\beta_{n}$ should be obtained from the Eq. (2.2), which obviously contains 
square roots of the coefficients (2.6). Eq. (2.8) being rewritten with the coefficients, $\alpha, \beta$, and $\gamma$, by using the formulas (2.6), also contains the square roots. The explanation is that: by substituting the expansions (3.1)-(3.3) into the system (2.2), (2.3) and Eq. (2.8) correspondingly, one gets for the sums in (3.1)-(3.3) the equations, which can be written with the integer powers of $\alpha, \beta$, and $\gamma$.

Proposition 2. There exist three special (doubly truncated) solutions of the system (2.2)-(2.3) which along with the corresponding functions $\zeta(t)(2.7)$, we denote as follows:

(1) $y_{0}(t), z_{0}(t)$, and $\zeta_{0}(t)$;

(2) $y_{+1}(t), z_{+1}(t)$, and $\zeta_{+1}(t)$; and

(3) $y_{-1}(t), z_{-1}(t)$, and $\zeta_{-1}(t)$.

These solutions are uniquely defined by their asymptotic expansions as $|t| \rightarrow+\infty$ :

$$
\begin{aligned}
& y_{k}(t)=y_{F}(t)+\mathcal{O}\left(t^{-\infty}\right), \\
& z_{k}(t)=z_{F}(t)+\mathcal{O}\left(t^{-\infty}\right), \\
& \zeta_{k}(t)=\zeta_{F}(t)+\mathcal{O}\left(t^{-\infty}\right)
\end{aligned}
$$

in the sectors

$$
-\pi+\pi k<\arg t<\pi+\pi k, \quad k=-1,0,+1 .
$$

Proof. The existence and uniqueness of the solutions for $\arg t=0$ is proved in our previous work [11] (see Sec. 3 of [11]). To prove the fact that these solutions can be analytically continued with the same asymptotics (3.4) into the whole sector (3.5) one should notice that for each asymptotic expansion (3.1)-(3.3) there is only one function analytic in the sector (3.5) with this asymptotics so that, it must coincide with the correspondent function: $y(t), z(t)$, or $\zeta(t)$, which has asymptotics (3.4) for $\arg t=0$.

Remark 2. By integrating Eq. (2.4) one finds that the corresponding functions $u_{k}(t)$ have the following asymptotic behavior :

$$
u_{k}(t)=\hat{u} e^{\frac{t}{2}}(1+o(1)), \quad k=-1,0,+1,
$$

where $\hat{u} \in \mathbb{C}\{0\}$ is the constant of integration. 
Proposition 3. The monodromy coordinates of the solutions, $y_{k}(t), z_{k}(t), u_{k}(t)$, and $\zeta_{k}(t)$ are as follows:

(1) $k=0 \Rightarrow m_{11}^{0}=0, m_{21}^{0}=\frac{2^{2 \Theta_{\infty}}}{\imath \hat{u}} e^{-\pi \imath \Theta_{\infty}}, m_{11}^{1}=0, m_{12}^{1}=\imath \hat{u} 2^{-2 \Theta_{\infty}}$,

$$
s_{2}=-2 \imath \hat{u} e^{\pi \imath \Theta_{\infty}} 2^{-2 \Theta_{\infty}} \cos \pi \Theta_{0}, \quad s_{1}=\frac{2 \imath}{\hat{u}} 2^{2 \Theta_{\infty}} \cos \pi \Theta_{1} ;
$$

(2) $k=+1 \Rightarrow m_{11}^{0}=2\left(\cos \pi \Theta_{0}+e^{-\pi \imath \Theta_{\infty}} \cos \pi \Theta_{1}\right), m_{21}^{0}=-\frac{\imath}{\hat{u}} e^{-\pi \imath \Theta_{\infty}} 2^{2 \Theta_{\infty}}$,

$$
\begin{aligned}
m_{11}^{1}=0, m_{12}^{1}=\imath 2^{-2 \Theta_{\infty}} \hat{u}, s_{2} & =2 \imath \hat{u} 2^{-2 \Theta_{\infty}} \cos \pi \Theta_{1}, \\
s_{1} & =-\frac{2 \imath}{\hat{u}} e^{\pi \imath \Theta_{\infty}} 2^{2 \Theta_{\infty}} \cos \pi \Theta_{0} ;
\end{aligned}
$$

(3) $k=-1 \Rightarrow m_{11}^{0}=0, m_{21}^{0}=\frac{2^{2 \Theta_{\infty}}}{\imath \hat{u}} e^{-\pi \imath \Theta_{\infty}}, m_{11}^{1}=2\left(\cos \pi \Theta_{1}+e^{-\pi \imath \Theta_{\infty}} \cos \pi \Theta_{0}\right)$,

$$
\begin{aligned}
m_{12}^{1}=\imath \hat{u} 2^{-2 \Theta_{\infty}}, s_{2} & =2 \imath \hat{u} 2^{-2 \Theta_{\infty}} e^{2 \pi \imath \Theta_{\infty}} \cos \pi \Theta_{1}, \\
s_{1} & =-\frac{2 \imath}{\hat{u}} 2^{2 \Theta_{\infty}} e^{-\pi \imath \Theta_{\infty}} \cos \pi \Theta_{0} .
\end{aligned}
$$

Proof. The formulas in item (1) were proved in Corollary 3.1 of [11]; the formulas in items (2) and (3) are the special cases of the ones obtained in Corollaries $3.1,3.2$ of [11].

Proposition 4. The following asymptotic formulas as $|t| \rightarrow+\infty$ and $k= \pm 1$ :

$$
\begin{aligned}
& y_{k}(t)=y_{0}(t)+2 \Xi_{k} \exp \left(k i \frac{\pi}{4}-\frac{i}{2} \arg t\right) \sqrt{\frac{2}{|t|}} e^{k \frac{i t}{2}}(1+o(1)), \\
& z_{k}(t)=z_{0}(t)+\frac{\Xi_{k}}{4} \exp \left(-k i \frac{\pi}{4}+\frac{i}{2} \arg t\right) \sqrt{\frac{|t|}{2}} e^{k \frac{i t}{2}}(1+o(1)), \\
& \zeta_{k}(t)=\zeta_{0}(t)+\frac{\Xi_{k}}{2} \exp \left(-k i \frac{\pi}{4}+\frac{i}{2} \arg t\right) \sqrt{\frac{|t|}{2}} e^{k \frac{i t}{2}}(1+o(1)),
\end{aligned}
$$

where

$$
\Xi_{k}=\frac{2}{\sqrt{2 \pi}}\left(\cos \left(\pi \Theta_{0}\right) e^{k \frac{\pi i \Theta_{\infty}}{2}}+\cos \left(\pi \Theta_{1}\right) e^{-k \frac{\pi i \Theta_{\infty}}{2}}\right),
$$

are valid for the doubly truncated solutions in the sectors:

$$
k=+1 \Rightarrow 0 \leq \arg t \leq \pi, k=-1 \Rightarrow-\pi \leq \arg t \leq 0 .
$$

Proof. For $\arg t=0, \pm \pi$ these asymptotic expansions follow from the Corollaries 3.1-3.3 of the paper [11] and Eqs. (2.9)-(2.12) of Sec. 2. Their extensions to the whole sectors (3.11) can be proved either by linearizing of the corresponding differential equations or by inspection that, being restricted on the solutions $y_{k}(t), z_{k}(t)$, and $\zeta_{k}(t)$, the proof of asymptotic expansions in [10], [11] is also valid for the complex $t$ in these sectors. 
Corollary 1. The (analytic continuation of the) solutions $y_{0}(t), z_{0}(t)$, and $\zeta_{0}(t)$ have asymptotic expansions (3.1)-(3.3) in the domain $-\pi<\arg t<+\infty$ and, in particular, $y_{+1}(t)=y_{0}(t), z_{+1}(t)=z_{0}(t)$, and $\zeta_{+1}(t)=\zeta_{0}(t)$ iff $\Xi_{+1}=0$. Analogously, the (analytic continuation of the) solutions $y_{0}(t), z_{0}(t)$, and $\zeta_{0}(t)$ have asymptotic expansions (3.1)-(3.3) in the domain $-\infty<\arg t<+\pi$ and, in particular: $y_{-1}(t)=y_{0}(t), z_{-1}(t)=z_{0}(t)$, and $\zeta_{-1}(t)=\zeta_{0}(t)$ iff $\Xi_{-1}=0$.

Proof. Induction on positive or negative $k \in \mathbb{Z}$ : $|\arg t-\pi k|<\pi$, by making use of Eqs. (2.9)-(2.12). The base of the induction follows from Proposition 4.

Corollary 2. The expansions (3.1)-(3.3) are convergent iff $\Xi_{+1}=\Xi_{-1}=0$.

Proof. The condition $\Xi_{+1}=\Xi_{-1}=0$ means that all the solutions $y_{k}(t), z_{k}(t)$, and $\zeta_{k}(t)$ are rational functions.

Corollary 3. The system (2.2), (2.3) and consequently the fifth Painlevé equation (2.5) have rational solutions with the property $\lim _{t \rightarrow \infty} y(t)=-1$, iff at least one of the following conditions:

$$
\begin{array}{ll}
\text { (1) } \Theta_{\infty}=2 l, & \Theta_{0}=\Theta_{1}+1+2 m \\
\text { (2) } \Theta_{\infty}=2 l, & \Theta_{0}=-\Theta_{1}+1+2 m, \\
\text { (3) } \Theta_{\infty}=1+2 l, & \Theta_{0}=\Theta_{1}+2 m \\
\text { (4) } \Theta_{\infty}=1+2 l, & \Theta_{0}=-\Theta_{1}+2 m \\
\text { (5) } \Theta_{0}=\frac{1}{2}+l, & \Theta_{1}=\frac{1}{2}+m,
\end{array}
$$

where $l, m \in \mathbb{Z}$, is satisfied. This solution is unique, if the coefficients of the system (2.2), (2.3) are set as shown above, and coincides with the doubly truncated solution, $\left(y_{0}(t), z_{0}(t)\right)$. In terms of the coefficients $\alpha, \beta$, and $\gamma(2.6)$ of the fifth Painlevé equation the conditions (1), (3) can be rewritten as follows:

$$
\sqrt{2 \alpha}=\frac{1}{2}+n_{1}, \quad \sqrt{-2 \beta}=\frac{1}{2}+n_{2}, \quad n_{1}, n_{2} \in \mathbb{Z}, \quad \gamma \in \mathbb{C},
$$

and the conditions (2), (4), (5) as:

$$
\sqrt{2 \alpha}+\sqrt{-2 \beta}=n_{1}, \quad \gamma=2 n_{2}-n_{1}, \quad n_{1}, n_{2} \in \mathbb{Z} .
$$

For the fixed set of $\alpha, \beta$, and $\gamma$ satisfying Eqs. (3.12) or/and Eqs. (3.13) for some choice of the branches of the square roots, this rational solution of the fifth Painlevé equation (2.5) is unique and coincides with $y_{0}(t)$. In this case the functions $z_{0}(t)$ and $\zeta_{0}(t)$ are also rational.

Proof. The proof follows from Corollary 2 and solution of the equation $\Xi_{-1}=$ $\Xi_{+1}$. 
Proposition 5. The solution of the system (2.2)-(2.4) with the following monodromy coordinates (the upper-truncated solution) :

$m_{11}^{0}=\sqrt{2 \pi} \hat{w} e^{-\frac{\pi \imath \Theta_{\infty}}{2}}, m_{21}^{0}=\frac{2^{2 \Theta_{\infty}}}{\imath u} e^{-\pi \imath \Theta_{\infty}}, s_{1}=\frac{2^{2 \Theta_{\infty}}}{\imath \hat{u}}\left(\sqrt{2 \pi} \hat{w} e^{\frac{\pi \imath \Theta_{\infty}}{2}}-2 \cos \pi \Theta_{1}\right)$,

$m_{11}^{1}=0, \quad m_{12}^{1}=\imath \hat{u} 2^{-2 \Theta_{\infty}}, \quad s_{2}=\imath \hat{u} 2^{-2 \Theta_{\infty}} e^{\pi \imath \Theta_{\infty}}\left(\sqrt{2 \pi} \hat{w} e^{-\frac{\pi \imath \Theta_{\infty}}{2}}-2 \cos \pi \Theta_{0}\right)$,

where $\hat{w} \in \mathbb{C}$, has in the sector $0 \leq \arg t \leq \pi$ the following asymptotic expansion as $|t| \rightarrow+\infty$ :

$$
\begin{aligned}
& y(t)=y_{0}(t)+2 \hat{w} \sqrt{\frac{2}{|t|}} e^{\frac{\imath}{2}\left(\frac{\pi}{2}-\arg t\right)} e^{\frac{\imath t}{2}}+\mathcal{O}\left(\frac{1}{t} e^{\frac{\imath t}{2}}\right), \\
& z(t)=z_{0}(t)+\frac{\hat{w}}{4} \sqrt{\frac{|t|}{2}} e^{-\frac{\imath}{2}\left(\frac{\pi}{2}-\arg t\right)} e^{\frac{\imath t}{2}}+\mathcal{O}\left(t^{-\frac{1}{2}} e^{\frac{\imath t}{2}}\right), \\
& u(t)=u_{0}(t)(1+o(1)), \\
& \zeta(t)=\zeta_{0}(t)+\frac{\hat{w}}{2} \sqrt{\frac{|t|}{2}} e^{\frac{\imath}{2}\left(\frac{\pi}{2}+\arg t\right)} e^{\frac{\imath t}{2}}+\mathcal{O}\left(t^{-\frac{1}{2}} e^{\frac{\imath t}{2}}\right) .
\end{aligned}
$$

Proof. The proof is based on the same arguments as the proof of Propositions 3 and 4.

Proposition 6. The solution of the system (2.2)-(2.4) with the following monodromy coordinates (the lower-truncated solution) :

$m_{11}^{0}=0, \quad m_{21}^{0}=\frac{2^{2 \Theta_{\infty}}}{\imath \hat{u}} e^{-\pi \imath \Theta_{\infty}}, \quad s_{2}=-\hat{u} 2^{-2 \Theta_{\infty}} e^{\pi \imath \Theta_{\infty}}\left(\sqrt{2 \pi} \hat{v} e^{\frac{\pi \imath \Theta_{\infty}}{2}}+2 \imath \cos \pi \Theta_{0}\right)$,

$m_{11}^{1}=\imath \sqrt{2 \pi} \hat{v} e^{-\frac{\pi \imath \Theta_{\infty}}{2}}, m_{12}^{1}=\imath \hat{u} 2^{-2 \Theta_{\infty}}, \quad s_{1}=\frac{2^{2 \Theta_{\infty}}}{\hat{u}}\left(\sqrt{2 \pi} \hat{v} e^{-\frac{\pi \imath \Theta_{\infty}}{2}}+2 \imath \cos \pi \Theta_{1}\right)$, where $\hat{v} \in \mathbb{C}$, has in the sector $-\pi \leq \arg t \leq 0$ the following asymptotic expansion as $|t| \rightarrow+\infty$ :

$$
\begin{aligned}
& y(t)=y_{0}(t)+2 \hat{v} \sqrt{\frac{2}{|t|}} e^{\frac{\imath}{2}\left(\frac{\pi}{2}-\arg t\right)} e^{-\frac{\imath t}{2}}+\mathcal{O}\left(\frac{1}{t} e^{-\frac{\imath t}{2}}\right) \\
& z(t)=z_{0}(t)-\frac{\hat{v}}{4} \sqrt{\frac{|t|}{2}} e^{-\frac{\imath}{2}\left(\frac{\pi}{2}-\arg t\right)} e^{-\frac{\imath t}{2}}+\mathcal{O}\left(t^{-\frac{1}{2}} e^{-\frac{\imath t}{2}}\right) \\
& u(t)=u_{0}(t)(1+o(1)) \\
& \zeta(t)=\zeta_{0}(t)+\frac{\hat{v}}{2} \sqrt{\frac{|t|}{2}} e^{\frac{\imath}{2}\left(\frac{\pi}{2}+\arg t\right)} e^{-\frac{\imath t}{2}}+\mathcal{O}\left(t^{-\frac{1}{2}} e^{-\frac{\imath t}{2}}\right) .
\end{aligned}
$$

Proof. The proof is based on the same arguments as the proof of Propositions 3 and 4 . 
Theorem 1. The polynomials: $\alpha_{n}\left(\Theta_{0}, \Theta_{1}, \Theta_{\infty}\right), \beta_{n}\left(\Theta_{0}, \Theta_{1}, \Theta_{\infty}\right)$, and $\gamma_{n}\left(\Theta_{0}, \Theta_{1}, \Theta_{\infty}\right)$ have the following asymptotics as $n \rightarrow \infty(\equiv p \rightarrow \infty)$ :

$$
\begin{aligned}
& \alpha_{2 p-1}\left(\Theta_{0}, \Theta_{1}, \Theta_{\infty}\right)=\mathcal{O}\left(C^{p}\right)+(-1)^{p} \frac{4^{2 p}}{2 \pi^{2}} \cos \frac{\pi \Theta_{\infty}}{2} \cos \frac{\pi}{2}\left(\Theta_{0}-\Theta_{1}\right) \times \\
& \cos \frac{\pi}{2}\left(\Theta_{0}+\Theta_{1}\right) \Gamma\left(p-\frac{3}{4}\right) \Gamma\left(p-\frac{1}{4}\right)\left(1+\mathcal{O}\left(\frac{1}{p}\right)\right), \\
& \alpha_{2 p}\left(\Theta_{0}, \Theta_{1}, \Theta_{\infty}\right)=\mathcal{O}\left(C^{p}\right)+(-1)^{p} \frac{4^{2 p+1}}{2 \pi^{2}} \sin \frac{\pi \Theta_{\infty}}{2} \sin \frac{\pi}{2}\left(\Theta_{0}-\Theta_{1}\right) \times \\
& \sin \frac{\pi}{2}\left(\Theta_{0}+\Theta_{1}\right) \Gamma\left(p-\frac{1}{4}\right) \Gamma\left(p+\frac{1}{4}\right)\left(1+\mathcal{O}\left(\frac{1}{p}\right)\right), \\
& \beta_{2 p-1}\left(\Theta_{0}, \Theta_{1}, \Theta_{\infty}\right)=\mathcal{O}\left(C^{p}\right)+(-1)^{p} \frac{4^{2 p-1}}{2 \pi^{2}} \cos \frac{\pi \Theta_{\infty}}{2} \cos \frac{\pi}{2}\left(\Theta_{0}-\Theta_{1}\right) \times \\
& \cos \frac{\pi}{2}\left(\Theta_{0}+\Theta_{1}\right) \Gamma\left(p-\frac{1}{4}\right) \Gamma\left(p+\frac{1}{4}\right)\left(1+\mathcal{O}\left(\frac{1}{p}\right)\right), \\
& \beta_{2 p}\left(\Theta_{0}, \Theta_{1}, \Theta_{\infty}\right)=\mathcal{O}\left(C^{p}\right)+(-1)^{p} \frac{4^{2 p}}{2 \pi^{2}} \sin \frac{\pi \Theta_{\infty}}{2} \sin \frac{\pi}{2}\left(\Theta_{0}-\Theta_{1}\right) \times \\
& \sin \frac{\pi}{2}\left(\Theta_{0}+\Theta_{1}\right) \Gamma\left(p+\frac{1}{4}\right) \Gamma\left(p+\frac{3}{4}\right)\left(1+\mathcal{O}\left(\frac{1}{p}\right)\right), \\
& \gamma_{2 p-1}\left(\Theta_{0}, \Theta_{1}, \Theta_{\infty}\right)=\mathcal{O}\left(C^{p}\right)+(-1)^{p} \frac{4^{2 p-1}}{\pi^{2}} \sin \frac{\pi \Theta_{\infty}}{2} \sin \frac{\pi}{2}\left(\Theta_{0}-\Theta_{1}\right) \times \\
& \sin \frac{\pi}{2}\left(\Theta_{0}+\Theta_{1}\right) \Gamma\left(p-\frac{1}{4}\right) \Gamma\left(p+\frac{1}{4}\right)\left(1+\mathcal{O}\left(\frac{1}{p}\right)\right), \\
& \gamma_{2 p}\left(\Theta_{0}, \Theta_{1}, \Theta_{\infty}\right)=\mathcal{O}\left(C^{p}\right)-(-1)^{p} \frac{4^{2 p}}{\pi^{2}} \cos \frac{\pi \Theta_{\infty}}{2} \cos \frac{\pi}{2}\left(\Theta_{0}-\Theta_{1}\right) \times \\
& \cos \frac{\pi}{2}\left(\Theta_{0}+\Theta_{1}\right) \Gamma\left(p+\frac{1}{4}\right) \Gamma\left(p+\frac{3}{4}\right)\left(1+\mathcal{O}\left(\frac{1}{p}\right)\right),
\end{aligned}
$$

where $\Gamma(\cdot)$ is the Gamma-function [13] and $C>0$ is independent of $p$.

Proof. The proof is given in the next Section.

Corollary 4. All leading terms of the above asymptotics vanish iff the parameters $\Theta_{0}, \Theta_{1}, \Theta_{\infty}$ satisfy one of the conditions of the Corollary 3. In this case, at least one: odd or even, subsequence of each sequence of the coefficients has the following asymptotic expansion:

$$
\begin{aligned}
& \left|\alpha_{n}\left(\Theta_{0}, \Theta_{1}, \Theta_{\infty}\right)\right| \underset{n \rightarrow \infty}{=} a_{\Theta_{0}, \Theta_{1}, \Theta_{\infty}}^{n}(1+o(1)), \\
& \left|\beta_{n}\left(\Theta_{0}, \Theta_{1}, \Theta_{\infty}\right)\right| \underset{n \rightarrow \infty}{=} b_{\Theta_{0}, \Theta_{1}, \Theta_{\infty}}^{n}(1+o(1)), \\
& \left|\gamma_{n}\left(\Theta_{0}, \Theta_{1}, \Theta_{\infty}\right)\right| \underset{n \rightarrow \infty}{=} c_{\Theta_{0}, \Theta_{1}, \Theta_{\infty}}^{n}(1+o(1)),
\end{aligned}
$$

where $a_{\Theta_{0}, \Theta_{1}, \Theta_{\infty}}, b_{\Theta_{0}, \Theta_{1}, \Theta_{\infty}}$, and $c_{\Theta_{0}, \Theta_{1}, \Theta_{\infty}}$ are the numbers equal to the maximums of absolute values of poles of the rational solutions to the system (2.2), 
(2.3) and Eq. (2.8), which are described in the Corollary 3. If the asymptotics of one of the subsequences is not given by the corresponding equation in (3.14), then the absolute values of its members (at least for a rather big $n$ ) are less than the r.-h.s. of one of the corresponding equation in (3.14).

Proof. The statement follows from Theorem 1 with the help of the Corollaries 2 and 3 .

\section{Derivation}

To derive the large $n$ asymptotics for the polynomials $\alpha_{n}\left(\Theta_{0}, \Theta_{1}, \Theta_{\infty}\right)$, $\beta_{n}\left(\Theta_{0}, \Theta_{1}, \Theta_{\infty}\right)$, and $\gamma_{n}\left(\Theta_{0}, \Theta_{1}, \Theta_{\infty}\right)$ (3.1)-(3.3), we use ideas based on the Borel transform. Here, we summarize some facts concerning the Borel transform, which we need for a derivation of the results stated in Theorem 1. The proofs of these results can be found in the monograph [12]. The following presentation is specific to the concrete problem under consideration.

Let $F(t)$ be one of the divergent series $y_{F}(t), z_{F}(t)$, or $\zeta_{F}(t)$. Let us denote the coefficients of these expansions as $f_{n}$, i.e.,

$$
F(t)=\sum f_{n} t^{-n}
$$

It is known that, the divergent expansions (3.1)-(3.3) for the systems like (2.2)(2.3) are Borel summable. In particular, in our case it means that for some positive numbers: $M, A$, and $a$, the following inequality is valid: $\left|f_{n} / \Gamma(a n+1)\right|<$ $M A^{-n}$, where $\Gamma(\cdot)$ is the $\Gamma$-function [13]. Actually, in our case $a=1$, but we use this fact only to make our derivation shorter: the fact which is really important for the following is that such number $a>0$ exists. The Borel transform, $B_{F}(p)$, is defined by the following, convergent in some neighborhood of $p=0$, series:

$$
B_{F}(p)=\sum \frac{f_{n}}{\Gamma(a n+1)} p^{n} .
$$

The analytic continuation of the function $B_{F}(p)$ into the sectors: $|\arg p+\pi k|<$ $\pi / 2, k=0, \pm 1(a=1$ !) has a finite exponential type $T$. The Laplace transform of $B_{F}(\tau / t)$ on $\tau$ is therefore convergent in the domain $|t|>T,|\arg t+\pi k|<\pi / 2$ and yields the function $f(t)$ (the Borel sum of $F(t)$ ):

$$
f(t)=\int_{0}^{\infty} d \tau e^{-\tau} B_{F}\left(t^{-1} \tau^{a}\right)
$$

which analytic continuation into the domain $|t|>T,|\arg t+\pi k|<\pi$ has the asymptotic expansion (4.1). Since analytic function with the asymptotic expansion (4.1) in the sector $|\arg t+\pi k|<\pi$ is unique, the function $\mathrm{f}(\mathrm{t})$ (4.3) coincide with the corresponding doubly truncated solution, $f_{k}(t)$, i.e., with one of the functions: $y_{k}(t), z_{k}(t)$, or $\zeta_{k}(t), k=0, \pm 1$.

The function $B_{F}(p)$ may have poles and cuts, running from its singularities to the infinity. The previous discussion shows that the only possibility for such singularities (and cuts) can be along the imaginary axis. The indications of these 
cuts are ruptures of the of the function $f(t)$ : in the sectors $|\arg t+\pi k|<\pi / 2$ this function coincides with $f_{k}(t)$. Thus in our case we have two such ruptures: along the positive and negative imaginary semi-axis. To get the asymptotic formulas announced in Theorem 1 for $f_{n}$, we proceed as follows:

$$
\begin{aligned}
& f_{n}=\Gamma(a n+1) \frac{f_{n}}{\Gamma(a n+1)}=\int_{0}^{+\infty} d \tau e^{-\tau} \tau^{a n} \frac{f_{n}}{\Gamma(a n+1)}=\frac{1}{2 \pi \imath} \int_{0}^{+\infty} d \tau e^{-\tau} \oint_{\Upsilon(\tau)} \frac{B_{F}\left(p \tau^{a}\right)}{p^{n+1}} d p \\
& =\left.\frac{1}{2 \pi \imath}\left(1+\mathcal{O}\left(\frac{1}{n}\right)\right) \int_{0}^{+\infty} d \tau e^{-\tau}\left(\int_{+i \infty}^{i A / \tau^{a}}+\int_{n \rightarrow \infty}^{-i \infty}\right) \frac{\Delta B_{F}\left(p \tau^{a}\right)}{p^{n+1}} d p\right|_{p=\frac{1}{i s}} \\
& =\frac{i^{n}}{2 \pi}\left(1+\mathcal{O}\left(\frac{1}{n}\right)\right) \int_{0}^{+\infty} d \tau e^{-\tau}\left(\int_{+0}^{\tau^{a} / A}+\int_{-\tau^{a} / A}^{-0}\right) s^{n-1} \Delta B_{F}\left(\frac{\tau^{a}}{i s}\right) d s \\
& =\frac{i^{n}}{2 \pi}\left(1+\mathcal{O}\left(\frac{1}{n}\right)\right)\left(\int_{+0}^{+\infty}+\int_{-i \infty}^{-0}\right) s^{n-1} d s \int_{0}^{+\infty} \Delta B_{F}\left(\frac{\tau^{a}}{i s}\right) e^{-\tau} d \tau,
\end{aligned}
$$

where $\Upsilon(\tau)$ is a positively oriented contour around 0 in the complex $p$-plane and $\Delta B_{F}(\cdot)$ is the difference of values of the Borel transform on the cut along the imaginary axis (r.h.s. minus l.h.s.). In the last integral we take into account that $\Delta B_{F}\left(\tau^{a} / i s\right)=0$ when $\left|\tau^{a}\right|$ is varying from 0 to $A s$. Finally, using Eq. (4.3) we arrive at the following asymptotic formula for $f_{n}$,

$$
\begin{aligned}
f_{n}=\underset{n \rightarrow \infty}{ } \mathcal{O}\left(C^{n}\right)+ & \\
\frac{\imath^{n-1}}{2 \pi}\left(1+\mathcal{O}\left(\frac{1}{n}\right)\right) & \left(\int _ { 0 } ^ { + \infty } s ^ { n - 1 } d s \left(f_{0}(\imath s)\right.\right. \\
& \left.-f_{+1}(\imath s)-(-1)^{n}\left(f_{0}(-\imath s)-f_{-1}(-\imath s)\right)\right),
\end{aligned}
$$

where $C>0$ and independent of $n$. The power error estimation in the last equation appear due to the fact that the integral representation (4.3) is valid only for $s>T$. Now, consequently substituting for $f_{k}$ in the last equation the asymptotic results from Proposition 4 for the doubly truncated solutions: $y_{k}(t), z_{k}(t)$, and $\zeta_{k}(t)$ defined in Proposition 2, we obtain the asymptotic expansions stated in the Theorem 1. Note, that by using the asymptotics in Eq. (4.4) instead of the exact solutions we get an additional error, which can be also estimated as $\mathcal{O}\left(C^{n}\right)$, where $C$ is independent of $n$. The error estimations indicated in the asymptotic expansions given in Proposition 4 are contributing to $\mathcal{O}(1 / p)$-correction terms for $f_{n}$ in Theorem 1.

\section{Appendix: Applications to the third Painlevé transcendent}

Here we consider two special cases of our results: the first one $\left(\Theta_{0}=\Theta_{1}=\right.$ $\left.\Theta_{\infty}=0\right)$ concerns the function $\sigma(x ; \lambda)$ which is discussed in the Sec. 1, the second example $\left(\Theta_{0}=\Theta_{1}=1 / 2, \Theta_{\infty}=0\right)$ shows, how one can reproduce one

of the first connection formulas for the Painlevé equations [7] (in our notations 
it is a connection formula for asymptotics of $P_{5}$ along the imaginary axis) by using the connection results for asymptotics on the real axis [10] and analytic continuation described in Sec. 3.

Proposition 7. A solution of the system (2.2), (2.3) with $\Theta_{0}=\Theta_{1}=\Theta_{\infty}=0$ is holomorphic at $t=0$ iff for some $z_{0} \in \mathbb{C} y(t)$ and $z(t)$ have the following asymptotic expansions as $t \rightarrow 0$ :

$$
\begin{aligned}
y(t)= & +t+\left(\frac{1}{2}-z_{0}\right) t^{2}+\left(\frac{1}{6}-z_{0}\right) t^{3}+\left(\frac{1}{4 !}-\frac{13}{24} z_{0}+\frac{z_{0}^{2}}{2}\right) t^{4} \\
& +\left(\frac{1}{5 !}-\frac{5}{24} z_{0}+\frac{5}{9} z_{0}^{2}\right) t^{5}+\left(\frac{1}{6 !}-\frac{137}{2160} z_{0}+\frac{25}{72} z_{0}^{2}-\frac{3}{16} z_{0}^{3}\right) t^{6} \\
& +\left(\frac{1}{7 !}-\frac{7}{432} z_{0}+\frac{847}{5400} z_{0}^{2}-\frac{35}{144} z_{0}^{3}\right) t^{7}+\left(\frac{z_{0}^{4}}{16}-\frac{19}{108} z_{0}^{3}+\frac{1}{8 !}\right. \\
& \left.-\frac{871}{241920} z_{0}+\frac{4907}{86400} z_{0}^{2}\right) t^{8}+\left(\frac{1}{9 !}-\frac{517}{725760} z_{0}+\frac{8189}{470400} z_{0}^{2}\right. \\
& \left.-\frac{497}{5400} z_{0}^{3}+\frac{3}{32} z_{0}^{4}\right) t^{9}+\left(\frac{1}{10 !}-\frac{1543}{12096000} z_{0}+\frac{989}{211680} z_{0}^{2}\right. \\
& \left.-\frac{48019}{1244160} z_{0}^{3}+\frac{805}{10368} z_{0}^{4}-\frac{5}{256} z_{0}^{5}\right) t^{10}+\mathcal{O}\left(t^{11}\right), \\
z(t)= & z_{0}+2 z_{0}^{2} t+3 z_{0}^{3} t^{2}+\left(\frac{z_{0}^{2}}{9}+4 z_{0}^{4}\right) t^{3}+\left(\frac{25}{144} z_{0}^{3}+5 z_{0}^{5}\right) t^{4} \\
& +\left(\frac{z_{0}^{2}}{300}+\frac{z_{0}^{4}}{4}+6 z_{0}^{6}\right) t^{5}+\left(\frac{49}{10800} z_{0}^{3}+\frac{49}{144} z_{0}^{5}+7 z_{0}^{7}\right) t^{6} \\
& +\left(\frac{1}{17640} z_{0}^{2}+\frac{121}{16200} z_{0}^{4}+\frac{4}{9} z_{0}^{6}+8 z_{0}^{8}\right) t^{7}+\left(\frac{761}{11289600} z_{0}^{3}\right. \\
& \left.+\frac{73}{6400} z_{0}^{5}+\frac{9}{16} z_{0}^{7}+9 z_{0}^{9}\right) t^{8}+\left(\frac{1}{1632960} z_{0}^{2}+\frac{1349}{9144576} z_{0}^{4}\right. \\
& \left.+\frac{19}{1152} z_{0}^{6}+\frac{25}{36} z_{0}^{8}+10 z_{0}^{10}\right) t^{9}+\left(\frac{7381}{11430720000} z_{0}^{3}\right. \\
& \left.+\frac{234377}{914457600} z_{0}^{5}+\frac{5929}{259200} z_{0}^{7}+\frac{121}{144} z_{0}^{9}+11 z_{0}^{11}\right) t^{10}+\mathcal{O}\left(t^{11}\right) .
\end{aligned}
$$

In that case expansions (A.1), (A.2), of course, coincide with the Taylor expansions of the functions $y(t)$ and $z(t)$ at $t=0$, moreover, the parameter $z_{0}$ characterize them uniquely.

Proof. By substituting formal power series for $y(t)$ and $z(t)$ into the system (2.2), (2.3) one gets the recursion relation which allow to uniquely determine all the coefficients as soon as $z_{0} \in \mathbb{C}$ is given and prove the convergence in the standard manner.

Proposition 8. In the case $\Theta_{0}=\Theta_{1}=\Theta_{\infty}=0$ the function $\zeta(t)(2.7)$ is holomorphic at $t=0$ iff the corresponding solution $(y(t), z(t))$ of the system (2.2), (2.3) is holomorphic at $t=0$. 
Proof. The proof follows from Proposition 7 and the equations:

$$
\zeta=-t z+\frac{z^{2}}{y}(1-y)^{2}, \quad z=-\zeta^{\prime}, \quad y=1+\frac{1}{2 z^{2}}\left(t z^{\prime}+\zeta+t z\right),
$$

where the prime denotes differentiation on $t$. Eqs. (A.3) are resulted from the system $(2.2),(2.3)$ and definition $(2.7)$. If $\zeta(t)$ is a holomorphic function at $t=0$, then according to the last equation in (A.3) the function $y(t)$ can, theoretically, have a pole at $t=0$ but this contradicts to the system $(2.2),(2.3)$.

Remark 3. It is easy to see that the coefficient, $z_{n}$, before $t^{n}$ in the Tailor series (A.2) is a polynomial of the power $n+1$ of the parameter $z_{0}$. For $n \geq 2$ the senior coefficients have the following structure: $z_{n}=(n+1) z_{0}^{n+1}+\frac{(n+1)^{2}}{3^{2} 2^{4}} z_{0}^{n}+\ldots$, while the structure of the junior terms is not evident. By denoting $y_{n}$ the coefficient before $t^{n}$ in the Tailor series (A.1): $y_{1}=1, y_{2}=1 / 2-z_{0}, \ldots$, we find (see Eq. (2.9)) that

$$
\sum_{k+l=m}(-)^{l} y_{l}\left(-z_{0}\right) y_{k}\left(z_{0}\right)+(-)^{m} y_{m}\left(-z_{0}\right)+y_{m}\left(z_{0}\right)=0 .
$$

The function $y(t)$ (A.1) can be presented in the form: $y(t)=e^{t}+z_{0} f\left(t, z_{0}\right)$, where the function $f\left(t, z_{0}\right)$ is holomorphic at $\left(t=0, z_{0}=0\right)$. The Tailor expansion for the function $\zeta(t)$ can be obtained by integration of the expansion (A.2), $\zeta(t)=-\int_{0}^{t} z(t) d t$. We write it in terms of the function $\sigma(x ; \lambda)$ (see Eq. (1.2)) by using the relation $\sigma(x ; \lambda)=\left.\zeta(0,0,0 ; 4 i x)\right|_{z_{0}=-i \lambda / 2}$ and introducing the parameter $\mu=2 \lambda$ :

$$
\begin{aligned}
\sigma\left(x ; \frac{\mu}{2}\right)= & -\mu x-\mu^{2} x^{2}-\mu^{3} x^{3}-\left(\mu^{4}-\frac{4}{9} \mu^{2}\right) x^{4}-\left(\mu^{5}-\frac{5}{9} \mu^{3}\right) x^{5} \\
& -\left(\mu^{6}-\frac{2}{3} \mu^{4}+\frac{32}{225} \mu^{2}\right) x^{6}-\left(\mu^{7}-\frac{7}{9} \mu^{5}+\frac{112}{675} \mu^{3}\right) x^{7} \\
& -\left(\mu^{8}-\frac{8}{9} \mu^{6}+\frac{484}{2025} \mu^{4}-\frac{64}{2205} \mu^{2}\right) x^{8}-\left(\mu^{9}-\mu^{7}\right. \\
& \left.+\frac{73}{225} \mu^{5}-\frac{3044}{99225} \mu^{3}\right) x^{9}-\left(\mu^{10}-\frac{10}{9} \mu^{8}+\frac{19}{45} \mu^{6}\right. \\
& \left.-\frac{10792}{178605} \mu^{4}+\frac{512}{127575} \mu^{2}\right) x^{10}-\left(\mu^{11}-\frac{11}{9} \mu^{9}+\frac{1078}{2025} \mu^{7}\right. \\
& \left.-\frac{85228}{893025} \mu^{5}+\frac{85888}{22325625} \mu^{3}\right) x^{11} \mathcal{O}\left(x^{12}\right) .
\end{aligned}
$$

Proposition 9. Solution of the system (2.2), (2.3) with $\Theta_{0}=\Theta_{1}=\Theta_{\infty}=0$ holomorphic at $t=0$ has the truncated behavior as $t \rightarrow \infty$ iff the parameter $z_{0}= \pm \frac{1}{2 \pi \imath}$. The solution with the expansions (A.1)-(A.2) with $z_{0}=\frac{1}{2 \pi \imath}$, $\zeta_{u}(0,0,0 ; t)$, is the upper-truncated (see Proposition 5) and can be characterized by the monodromy coordinates:

$$
m_{11}^{0}=2, \quad m_{21}^{0}=(i u)^{-1}, m_{11}^{1}=0, m_{12}^{1}=i u, s_{1}=s_{2}=0 .
$$


The solution with the expansions (A.1)-(A.2) with $z_{0}=-\frac{1}{2 \pi \imath}, \zeta_{l}(0,0,0 ; t)$, is the lower-truncated (see Proposition 6) and can be characterized by the monodromy coordinates:

$$
m_{11}^{0}=0, \quad m_{21}^{0}=(i u)^{-1}, \quad m_{11}^{1}=2, \quad m_{12}^{1}=i u, \quad s_{1}=s_{2}=0
$$

Proof. The assertion of the proposition follows from our previous work [10], see Theorems 4.1-4.3, where the monodromy coordinates of the general solution of the system (2.2)-(2.4) were calculated. In these theorems the small t asymptotics of the general solution of the system (2.2)-(2.4) is characterized by the complex parameters $\sigma$ and $\hat{z}$. From the Theorems 4.1 and 4.3 we see that the solution can be holomorphic at $t=0$ only if the parameter $\sigma=0$ (in the case $\sigma=1$ one of the functions $y(t)$ or $z(t)$ has a pole at the origin. Put in the formulas (4.1) of the work [10] $\Theta_{0}=\Theta_{1}=\Theta_{\infty}=0$ and than $\sigma \rightarrow 0$ to find that $\hat{s} \underset{\sigma \rightarrow 0}{=} \sigma /\left(8 z_{0}\right)$. On the other hand Theorem 4.2 shows that in the case $\Theta_{0}=\Theta_{1}=\Theta_{\infty}=\sigma=0$ the Stokes multipliers $s_{1}=s_{2}=0$ and the limits as $t \rightarrow 0$ of $m_{11}^{0}=1-2 \pi \imath z_{0}, m_{22}^{0}=$ $1+2 \pi \imath z_{0}$. Comparing these formulas with the monodromy coordinates of the upper/lower-truncated solutions (see Propositions 5,6) we get that necessarily $z_{0}= \pm 1 /(2 \pi \imath)$. Finally, calculating the remaining monodromy coordinates we arrive at the Eqs. (A.5)-(A.6).

Corollary 5. For $\lambda=1 / \pi$ the function $\sigma(x ; \lambda)$ defined by Eqs. (1.1), (1.2) satisfies the following equality:

$$
\sigma\left(x ; \frac{1}{\pi}\right)=\zeta_{u}(0,0,0 ; 4 i x)=\zeta_{l}(0,0,0 ;-4 i x),
$$

where $\zeta_{u}(0,0,0 ; \cdot)$ and $\zeta_{l}(0,0,0 ; \cdot)$ are the solutions of the Eq. (2.8) which are specified in Proposition 9. Asymptotics of the sequence $c_{n}$ (see Eq. (1.3)) is as follows:

$$
c_{n}=-\frac{\gamma_{2 n}(0,0,0)}{(4 i)^{2 n}} \underset{n \rightarrow \infty}{=} \frac{1}{\pi^{2}} \Gamma\left(n+\frac{1}{4}\right) \Gamma\left(n+\frac{3}{4}\right)\left(1+\mathcal{O}\left(\frac{1}{n}\right)\right) .
$$

Proof. The first equality in (A.7) follows from the definition of $\sigma(x ; \lambda)$ and Proposition 9. The second equality resulted from the Proposition 9 and formulas (2.10)-(2.12). The equation (A.8) is the special case of the last formula in Theorem 1.

Remark 4. Since (see [13]) we have

$$
\Gamma\left(n+\frac{1}{4}\right) \Gamma\left(n+\frac{3}{4}\right) \underset{n \rightarrow \infty}{=} n !(n-1) !\left(1-\frac{0,1875}{n}+\mathcal{O}\left(\frac{1}{n^{2}}\right)\right),
$$

the Eq. (A.8) proves the conjecture (1.5) by E. L. Basor and C. A. Tracy. More exactly we can write:

$$
c_{n} \underset{n \rightarrow \infty}{=} \frac{1}{\pi^{2}} n !(n-1) !\left(1+\mathcal{O}\left(\frac{1}{n}\right)\right) .
$$


Although formally the error estimations in the formulas (A.8) and (A.10) are the same, the leading term in our formula (A.8) more exactly approximates the numbers $c_{n}$, since the term $\frac{3}{16 n}$ in Eq. (A.9) add the systematic error equal to $\frac{3}{16 \pi^{2}}(n-1) !^{2}$. In the last two columns of the following table we present the results of calculations of the numbers $c_{n}$ for $n=5,10$, and 15 via the leading terms of asymptotic expansions (A.8) and (A.10) correspondingly.

\begin{tabular}{|l|l|l|l|}
\hline$c_{n}$ & Exact value & Eq.(A.8) & Eq.(A.10) \\
\hline$c_{5}$ & $\frac{1080091}{4096}=263.694091796875$ & $281.0779 \ldots$ & $291.8050 \ldots$ \\
\hline$c_{10}$ & $\frac{266126511253407125}{2097152}=0.12689 \ldots \cdot 10^{12}$ & $0.1309 \ldots \cdot 10^{12}$ & $0.1334 \ldots \cdot 10^{12}$ \\
\hline$c_{15}$ & $\frac{23990916462228670446948154489421}{2147483648}=$ & $0.1140 \ldots \cdot 10^{23}$ & $0.1155 \ldots \cdot 10^{23}$ \\
& $0.1117 \ldots \cdot 10^{23}$ & & \\
\hline
\end{tabular}

Our numerical calculations show that the error term in Eq. (A.8) can be evaluated as $\approx-0,3105 / n$. To calculate it explicitly or, more generally, to develop the asymptotic expansions obtained in the Theorem 1 into the complete asymptotic series, one has to study the so-called trans-series for the system $(2.2),(2.3)$ and Eq. (2.8).

Proposition 10. If $\Theta_{0}=\Theta_{1}=1 / 2, \Theta_{\infty}=0$, then the upper-truncated solutions, $y(i \tau)$, (see Proposition 5) with the parameter $\hat{w}=\frac{2 i}{\sqrt{2 \pi}} \sin \frac{\pi \sigma}{2}$, where $0<\Re \sigma<1$, have the following asymptotics:

$$
\begin{gathered}
y(i \tau) \underset{\tau \rightarrow+0}{=} 1+4 i K\left(\frac{\tau}{2}\right)^{\sigma}+\mathcal{O}\left(\tau^{2 \sigma}\right)+\mathcal{O}(\tau), \\
y(i \tau) \underset{\tau \rightarrow+\infty}{=}-1-\frac{4 i \sin \frac{\pi \sigma}{2}}{\sqrt{\pi \tau}} e^{-\frac{\tau}{2}}\left(1+\mathcal{O}\left(\frac{e^{-\frac{\tau}{2}}}{\sqrt{\tau}}\right)\right), \\
z(i \tau) \underset{\tau \rightarrow+0}{=} \frac{(1+\sigma)}{8} i K\left(\frac{\tau}{2}\right)^{\sigma}-\frac{1}{4}+\frac{(1-\sigma)}{8 i K}\left(\frac{\tau}{2}\right)^{-\sigma}+\mathcal{O}\left(\tau^{1-\sigma}\right), \\
\zeta(i \tau) \underset{\tau \rightarrow+0}{=} \frac{\sigma^{2}}{4}-\frac{i s}{8}\left((i K)^{\frac{1}{2}}\left(\frac{\tau}{2}\right)^{\frac{\sigma}{2}}-(i K)^{-\frac{1}{2}}\left(\frac{\tau}{2}\right)^{-\frac{\sigma}{2}}\right)^{2}+\mathcal{O}\left(\tau^{2-2 \sigma}\right), \\
\text { where } K=2^{-3 \sigma} \frac{\Gamma\left(\frac{1}{2}-\frac{\sigma}{2}\right)}{\Gamma\left(\frac{1}{2}+\frac{\sigma}{2}\right)} .
\end{gathered}
$$

Proof. These results are the consequence of the Theorems 4.1 and 4.2 of our previous work [10]. More exactly, one has to put $\Theta_{0}=\Theta_{1}=1 / 2$ and $\Theta_{\infty}=0$ in the formulas (4.3) and (4.4) of Theorem 4.2: this considerably simplifies them, in particular, parameters $\hat{m}_{11}^{p}=\hat{m}_{22}^{p}=0$. After that, by equating the monodromy coordinates of the upper-truncated solutions given in Proposition 5 with the ones given in Theorem 4.2, one finds the relations between the parameter $w$, which defines the asymptotics as $t \rightarrow \infty$ and the parameters $\sigma$ and $\hat{s}$ (see formulas (4.1) and (4.2) of [10]), which define asymptotics of the general solutions of the system (2.2) and (2.3) as $t \rightarrow 0$. More exactly, one gets the equations relating 
$w$ with $\sigma$ (see above) and $\hat{u}$ with $r: \hat{u}=i r$, where the parameter $r$ defines asymptotics of the function $u(t)$ (see Eq. (2.4)): $u(t) \underset{t \rightarrow 0}{=}-r\left(1+\mathcal{O}\left(t^{\sigma}\right)\right.$ ), by comparing formulas for the Stokes multipliers, $s_{1}, s_{2}$. All the other equations are equivalent to $(\sigma-1) \hat{s}=i(1+\sigma) K$, where $K$ is given above. Finally, one finds the asymptotics of the functions $y(t), z(t)$, and $\zeta(t)$ as $t \rightarrow 0$ by substituting $\hat{s}$ into the Eqs. (4.1) and (4.2) of the work [10].

Corollary 6. [7] For each $\sigma \in \mathbb{C}, 0<\Re \sigma<1$, there exists a unique solution, $\eta=\eta(\tau)$, of the third Painlevé equation,

$$
\eta^{\prime \prime}=\frac{\eta^{\prime 2}}{\eta}-\frac{\eta^{\prime}}{\tau}+\frac{1}{16}\left(\eta^{3}-\eta^{-1}\right)
$$

where the primes denote differentiation on $\tau$, with the following asymptotics:

$$
\begin{aligned}
& \eta(\tau) \underset{\tau \rightarrow+0}{=} K\left(\frac{\tau}{2}\right)^{\sigma}+\mathcal{O}\left(\tau^{2 \sigma}\right)+\mathcal{O}(\tau), \\
& \eta(\tau) \underset{\tau \rightarrow+\infty}{=} 1-\frac{2 \sin \frac{\pi \sigma}{2}}{\sqrt{\pi \tau}} e^{-\frac{\tau}{2}}\left(1+\mathcal{O}\left(\frac{e^{-\frac{\tau}{2}}}{\sqrt{\tau}}\right)\right),
\end{aligned}
$$

in which $K$ is given in Proposition 10.

Proof. $\quad \eta(\tau)=-i \frac{\sqrt{y(i \tau)}-1}{\sqrt{y(i \tau)}+1}$

\section{Acknowledgement}

A.V.K. is grateful to L. Vinet and P. Winternitz for the invitation to the workshop "Theory of Nonlinear Special Functions: The Painlevé Transcendents" (May 13-18, 1996) in CRM Université de Montréal, where these results were announced, and P. M. Bleher and A. R. Its for the invitation to the joint AMSSIAM-IMS summer research conference "Random Matrices, Statistical Mechanics, and Painlevé Transcendents" (June 23-27, 1996) in Mount Holyoke, where this work was presented.

\section{References}

1. E. L. Basor and C. A. Tracy, Some problems associated with the asymptotics of $\tau$-functions, Preprint RIMS-845, Kyoto University, November 1991.

2. M. L. Mehta, Random matrices, 2nd edition, Academic Press, San Diego (1991).

3. C. A. Tracy and H. Widom, Introduction to random matrices, In: Helminck, G. F. (ed.), Geometric and quantum aspects of integrable systems. Proceedings of the eighth Scheveningen conference, Scheveningen, The Netherlands, August 16-21, 1992. Berlin: Springer-Verlag, (ISBN 3- 540-57365-8/hbk), Lect. Notes Phys. 424, (1993), 103-130; hep-th 9210073.

4. B. M. McCoy and Sh. Tang, Connection formulae for Painlevé V functions, Physica 19D (1986), 42-72; 20D (1986), 187-216.

5. L. A. Bordag and A. V. Kitaev, Algebraic and rational solutions of the fifth Painlevé equation (Russian), Comm. JINR, Dubna, (1986).

6. A. V. Kitaev, C. K. Law, and J. B. McLeod, Rational solutions of the fifth Painlevé equation, DIE 7 (1994), 967-1000. 
7. B. M. McCoy, C. A. Tracy, and T. T. Wu, Painlevé functions of the third kind, J. Math. Phys. 18 (1977), 1058-1092.

8. M. Jimbo and T. Miwa, Monodromy preserving deformation of linear ordinary differential equations with rational coefficients II, Physica 2D (1981), 407-448.

9. K. Okamoto, On the $\tau$-function of the Painlevé equations, Physica 2D (1981), 525-535.

10. F. V. Andreev and A. V. Kitaev, Connection formulas for the asymptotics of the fifth Painlevé transcendent on the real axis I, Sfb 288 Preprint 160, TU-Berlin (1995). http://www-sfb288.math.tu-berlin.de

11. Connection formulas for the asymptotics of the fifth Painleve transcendent on the real axis II, Sfb 288 Preprint 196, TU-Berlin (1996). http://www-sfb288.math.tu-berlin.de

12. G. Doetsch, Handbuch der Laplace-Transformation I, II, Birkhäuser Verlag Basel (1950), (1955).

13. H. Bateman and A. Erdélyi, Higher transcendental functions, I, II, McGraw-Hill Book Company Inc. (1953).

Steklov Mathematical Institute Fontanka 27, St. Petersburg, 191011, RUSSiA E-mail address: andreev@pdmi.ras.ru, kitaev@pdmi.ras.ru 